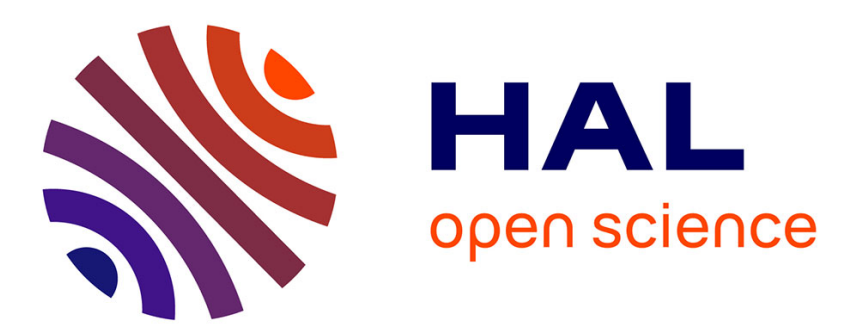

\title{
Approach to a stationary state in an external field
}

Angel Alastuey, Jaroslaw Piasecki

\section{To cite this version:}

Angel Alastuey, Jaroslaw Piasecki. Approach to a stationary state in an external field. Journal of Statistical Physics, 2010, 139, pp.991-1012. 10.107/s10955-010-9976-x . hal-00461276

\section{HAL Id: hal-00461276 https://hal.science/hal-00461276}

Submitted on 4 Mar 2010

HAL is a multi-disciplinary open access archive for the deposit and dissemination of scientific research documents, whether they are published or not. The documents may come from teaching and research institutions in France or abroad, or from public or private research centers.
L'archive ouverte pluridisciplinaire HAL, est destinée au dépôt et à la diffusion de documents scientifiques de niveau recherche, publiés ou non, émanant des établissements d'enseignement et de recherche français ou étrangers, des laboratoires publics ou privés. 


\title{
APPROACH TO A STATIONARY STATE IN AN EXTERNAL FIELD
}

\author{
A.Alastuey* J.Piasecki** \\ * ENS Lyon, CNRS, France \\ **Institute of Theoretical Physics, \\ University of Warsaw, Hoża 69, \\ 00681 Warsaw, Poland
}

(Dated: March 4, 2010)

\begin{abstract}
We study relaxation towards a stationary out of equilibrium state by analyzing a onedimensional stochastic process followed by a particle accelerated by an external field and propagating through a thermal bath. The effect of collisions is described within Boltzmann's kinetic theory. We present analytical solutions for the Maxwell gas and for the very hard particle model. The exponentially fast relaxation of the velocity distribution toward the stationary form is demonstrated. In the reference frame moving with constant drift velocity the hydrodynamic diffusive mode is shown to govern the distribution in the position space. We show that the exact value of the diffusion coefficient for any value of the field is correctly predicted by the Green-Kubo autocorrelation formula generalized to the stationary state.

PACS numbers: 05.30.-d, 05.70.Ce, 52.25.Kn
\end{abstract}




\section{INTRODUCTION}

The present paper is devoted to the the study of a stochastic process followed by a particle moving through a scattering thermal bath while accelerated by an external field. The field prevents the particle from acquiring the Maxwell distribution of the bath. Our aim here is not only to establish the precise form of the stationary velocity distribution, as it was e.g. the case in the analysis presented in [1], but also to answer the physically relevant question of the dynamics of approach towards the long-time asymptotic state. The evolution of the distribution in position space will be thus also discussed.

We consider a one-dimensional dynamics described by the Boltzmann kinetic equation

$$
\left(\frac{\partial}{\partial t}+v \frac{\partial}{\partial r}+a \frac{\partial}{\partial v}\right) f(r, v ; t)=v_{\text {int }}^{1-\gamma} \rho \int \mathrm{d} w|v-w|^{\gamma}[f(r, w ; t) \phi(v)-f(r, v ; t) \phi(w)]
$$

Here $f(r, v ; t)$ is the probability density for finding the propagating particle at point $r$ with velocity $v$ at time $t$. The thermal bath particles are not coupled to the external field. Before binary encounters with the accelerated particle they are assumed to be in an equilibrium state with uniform temperature $T$ and density $\rho$

$$
\rho \phi(v)=\rho \sqrt{\frac{m}{2 \pi k_{B} T}} \exp \left(-\frac{m v^{2}}{2 k_{B} T}\right)=\frac{\rho}{v_{\text {th }} \sqrt{2 \pi}} \exp \left[-\frac{1}{2}\left(\frac{v}{v_{\text {th }}}\right)^{2}\right]
$$

Here $\phi(v)$ is the Maxwell distribution, and

$$
v_{\mathrm{th}}=\sqrt{\frac{k_{B} T}{m}}
$$

denotes the corresponding thermal velocity. The differential operator on the lefthand side of (1) generates motion with a constant acceleration $a$. The accelerated motion is permanently perturbed by instantaneous exchanges of velocities with thermalized bath particles. This is modeled by the Boltzmann collision term on the right hand side of equation (1), which accounts for elastic encounters between equal mass particles. The collision frequency depends therein on the absolute relative velocity $|v-w|$ through a simple power law with exponent $\gamma$. Finally $v_{\text {int }}$ is some characteristic velocity of the underlying interparticle interaction. 
In the case of hard rods $(\gamma=1)$ the factor $|v-w|$ is the main source of difficulties in the attemps to rigorously determine the evolution of $f(r, v ; t)$, since it prevents the effective use of Laplace and Fourier transformations. It was thus quite remarkable that a stationary velocity distribution could be analytically determined in that case, leading in particular to an explicit expression for the current at any value of the external acceleration [1]. In that case, kinetic equation (11) has been solved exactly only at zero temperature where $\left.\phi(v)\right|_{T=0}=\delta(v)$ [2]. Also, when $\phi(v)$ is replaced by the distribution $\left[\delta\left(v-v_{0}\right)+\delta\left(v+v_{0}\right)\right] / 2$ with a discrete velocity spectrum $\pm v_{0}$, an explicit analytic solution has been derived and analyzed in [3] and [4]. The physically relevant conclusions from those works can be summarized as follows

(i) the approach to the asymptotic stationary velocity distribution is exponentially fast

(ii) in the reference system moving with average velocity, the hydrodynamic diffusion mode governs the spreading of the distribution in position space

(iii) the Green-Kubo autocorrelation formula for the diffusion coefficient applies in the non-equilibrium steady state

Our aim is to show that the general features (i)-(iii) persist when $\phi(v)$ is the Maxwell distribution with temperature $T>0$. However, in the present study, we restrict the analysis to cases $\gamma=0$ and $\gamma=2$, which are much simpler than the hard-rod one. Indeed, it turns out that the Fourier-Laplace transformation can then be effectively used to solve the initial value problem for equation (1). The simplifications occuring when $\gamma=0$ or $\gamma=2$ have been already exploited in other studies: for recent applications to granular fluids, see e.g. [5]-[7] and references quoted therein.

In terms of dimensionless variables

$$
w=v / v_{\text {th }}, \quad x=r \rho\left(v_{\text {th }} / v_{\text {int }}\right)^{\gamma-1}, \quad \tau=t \rho v_{\text {th }}\left(v_{\text {th }} / v_{\text {int }}\right)^{\gamma-1}
$$

the kinetic equation (II) takes the form

$$
\left(\frac{\partial}{\partial \tau}+w \frac{\partial}{\partial x}+\epsilon \frac{\partial}{\partial w}\right) F(x, w ; \tau)=\int \mathrm{d} u|w-u|^{\gamma}[F(x, u ; \tau) \Phi(w)-F(x, w ; \tau) \Phi(u)],
$$


where $\Phi(w)$ is the dimensionless normalized gaussian

$$
\Phi(w)=\frac{1}{\sqrt{2 \pi}} e^{-w^{2} / 2},
$$

and $\epsilon$ is the dimensionless parameter

$$
\epsilon=\left(v_{\text {th }} / v_{\text {int }}\right)^{1-\gamma} \frac{a m \rho^{-1}}{k_{B} T}
$$

proportional to the ratio between the energy $a m \rho^{-1}$ provided to the particle on a mean free path, and thermal energy $k_{B} T$. That parameter can thus be looked upon as a measure of the strength of the field. Integration of (5) over the position space yields the kinetic equation for the velocity distribution

$$
G(w ; \tau)=\int \mathrm{d} x F(x, w ; \tau)
$$

which reads

$$
\left(\frac{\partial}{\partial \tau}+\epsilon \frac{\partial}{\partial w}\right) G(w ; \tau)=\int \mathrm{d} u|w-u|^{\gamma}[G(u ; \tau) \Phi(w)-G(w ; \tau) \Phi(u)] .
$$

The paper is organized as follows. In Section II, we consider the so-called Maxwell gas $(\gamma=0)$. The explicit solution of the kinetic equation (5) enables a thorough discussion of the approach to the stationary state, together with a study of the structure of the stationary velocity distribution. In Section III, we proceed to a similar analysis for the very hard particle model $(\gamma=2)$. Section IV contains conclusions. Some calculations have been relegated to Appendices.

\section{THE MAXWELL GAS}

We consider here the simple version $\gamma=0$ of equation (5). One usually then refers to the Maxwell gas dynamics, in which the collision frequency does not depend on the speed of approach (see e.g. [8]). This case can be viewed upon as a very crude approximation to the hard rod dynamics $(\gamma=1)$ obtained by replacing the relative speed $|v-c|$ of colliding particles by constant thermal velocity $v_{\text {th }}$, while $v_{\text {int }}$ is identified with $v_{\text {th }}$. Here, kinetic equation (5) takes the form

$$
\begin{aligned}
\left(\frac{\partial}{\partial \tau}+w \frac{\partial}{\partial x}+\epsilon \frac{\partial}{\partial w}\right) F(x, w ; \tau) & =\int \mathrm{d} u[F(x, u ; \tau) \Phi(w)-F(x, w ; \tau) \Phi(u)] \\
& =M_{0}(x ; \tau) \Phi(w)-F(x, w ; \tau)
\end{aligned}
$$


where $M_{0}(x ; \tau)$ denotes the zeroth moment

$$
M_{0}(x ; \tau)=\int \mathrm{d} u F(x, u ; \tau) .
$$

Equation (9) can be conveniently rewritten as an integral equation

$$
\begin{aligned}
F(x, w ; \tau)=e^{-\tau} F(x-w \tau & \left.+\epsilon \tau^{2} / 2, w-\epsilon \tau ; 0\right) \\
& +\int_{0}^{\tau} d \eta e^{-\eta} \Phi(w-\epsilon \eta) M_{0}\left(x-w \eta+\epsilon \eta^{2} / 2 ; \tau-\eta\right),
\end{aligned}
$$

with an explicit dependence on the initial condition $F(x, w ; 0)$. Integration of equation (11) over $x$ yields

$$
G(w ; \tau)=\int \mathrm{d} x F(x, w ; \tau)=e^{-\tau} G_{\text {in }}(w-\epsilon \tau)+N_{0} \int_{0}^{\tau} \mathrm{d} \eta e^{-\eta} \Phi(w-\epsilon \eta),
$$

where $G_{\text {in }}(w)=G(w ; 0)$ is the initial condition, and $N_{0}=\int \mathrm{d} w \int \mathrm{d} x F(x, w ; \tau)=$ $\int \mathrm{d} w G(w ; \tau)$ is the conserved normalization factor.

\section{A. Stationary solution and relaxation of the velocity distribution}

Putting $N_{0}=1$ in formula (12) yields the evolution law for the normalized velocity distribution

$$
G(w ; \tau)=\int \mathrm{d} x F(x, w ; \tau)=e^{-\tau} G_{\text {in }}(w-\epsilon \tau)+\int_{0}^{\tau} \mathrm{d} \eta e^{-\eta} \Phi(w-\epsilon \eta),
$$

The first term on the right hand side of (13) describes the decaying memory of the initial distribution : $G_{\text {in }}(w)$ propagates in the direction of the field with constant velocity $\epsilon$, while its amplitude is exponentially damped. Clearly, for times $\tau \gg 1$ that term can be neglected.

The second term in formula (13) describes the approach to the asymptotic stationary distribution

$$
\begin{aligned}
G_{\mathrm{st}}(w)=G(w ; \infty) & =\int_{0}^{\infty} \mathrm{d} \eta e^{-\eta} \Phi(w-\epsilon \eta) \\
& =\frac{1}{2 \epsilon} \exp \left(\frac{1}{2 \epsilon^{2}}-\frac{w}{\epsilon}\right)\left(1+\operatorname{Erf}\left(\frac{w \epsilon-1}{\epsilon \sqrt{2}}\right)\right)
\end{aligned}
$$

where

$$
\operatorname{Erf}(\xi)=\frac{2}{\sqrt{\pi}} \int_{0}^{\xi} \mathrm{d} u \exp \left(-u^{2}\right)
$$




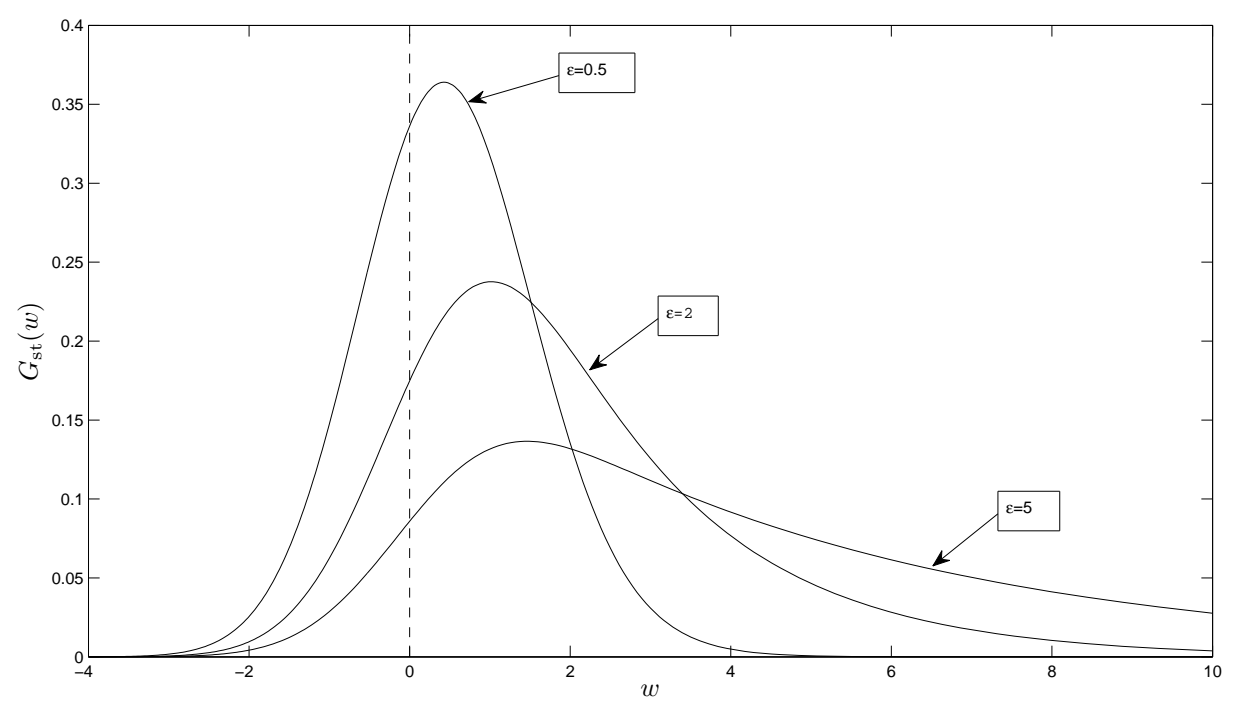

FIG. 1: Stationary velocity distribution $G_{\mathrm{st}}(w)$ for three values of $\epsilon$.

is the familiar error function. It is interesting to compare the decay-law of $G_{\mathrm{st}}(w)$ at large velocities, to that corresponding to the case of hard-rod collisions. Using expression (14) we find the asymptotic formula

$$
G_{\mathrm{st}}(w) \sim \frac{1}{\epsilon} \exp \left(\frac{1}{2 \epsilon^{2}}-\frac{w}{\epsilon}\right)
$$

when $w \rightarrow+\infty$. In contradistinction to the hard-rod case governed by an $\epsilon$ dependent gaussian law (see [1]) we find here a purely exponential decay. The thermal bath is unable to impose via collisions its own gaussian decay because of insufficient collision frequency. The replacement of the relative speed in the Boltzmann collision operator by thermal velocity implies thus qualitative changes in the shape of the stationary velocity distribution. The plot of $G_{\mathrm{st}}(w)$ for different values of $\epsilon$ is shown in Fig. 11.

Basic properties (i)-(iii) discussed in the Introduction turn out to be valid. Indeed, the inequality

$$
G_{\mathrm{st}}(w)-\int_{0}^{\tau} \mathrm{d} \eta e^{-\eta} \Phi(w-\epsilon \eta)=\int_{\tau}^{\infty} \mathrm{d} \eta e^{-\eta} \Phi(w-\epsilon \eta)<\frac{e^{-\tau}}{\epsilon}
$$

displays an uniform exponentially fast approach towards the stationary state. In particular, using formula (13), we find that the average velocity $<w>(\tau)$ approaches the asymptotic value

$$
<w>_{\text {st }}=\epsilon
$$


according to

$$
<w>(\tau)=\int \mathrm{d} w w G(w ; \tau)=\epsilon+e^{-\tau}\left[<w>_{\text {in }}-\epsilon\right]
$$

We encounter here an exceptional situation where the linear response is exact for any value of the external field.

Equation (12) with $N_{0}$ put equal to zero can be used for the evaluation of the time-displaced velocity autocorrelation function

$$
\Gamma(\tau)=<\left[w(\tau)-<w>_{\text {st }}\right]\left[w(0)-<w>_{\text {st }}\right]>_{\text {st }} .
$$

where $\langle\ldots\rangle_{\text {st }}$ denotes the average over stationary state (14). The calculation presented in Appendix B provides the formula

$$
\Gamma(\tau)=e^{-\tau}\left[1+\epsilon^{2}\right]
$$

which yields a remarkably simple field dependence of the diffusion coefficient

$$
D(\epsilon)=\int_{0}^{\infty} \mathrm{d} \tau \Gamma(\tau)=1+\epsilon^{2} .
$$

\section{B. Relaxation of density: appearence of a hydrodynamic mode}

Let us turn now to the analysis of the evolution of the normalized density $n(x ; \tau)=M_{0}(x ; \tau)$ in position space. It turns out that one can solve the complete integral equation (11) by applying to both sides Fourier and Laplace transformations. If we set

$$
\tilde{F}(k, w ; z)=\int_{0}^{\infty} \mathrm{d} \tau e^{-z \tau} \int \mathrm{d} x e^{-i k x} F(x, w ; \tau),
$$

we find

$$
\begin{array}{r}
\tilde{F}(k, w ; z)=\int_{0}^{\infty} \mathrm{d} \tau \exp \left[-i k\left(w \tau-\epsilon \frac{\tau^{2}}{2}\right)-(z+1) \tau\right] \\
\left\{\hat{F}_{\text {in }}(k, w-\epsilon \tau)+\tilde{n}(k ; z) \Phi(w-\epsilon \tau)\right\},
\end{array}
$$

where $\tilde{n}(k ; z)$ is the Fourier-Laplace transform of $n(x ; \tau)$, and

$$
\hat{F}_{\text {in }}(k, w)=\int \mathrm{d} x e^{-i k x} F(x, w ; 0)
$$


denotes the spatial Fourier transform of the initial condition. Equation (23) when integrated over the velocity space yields the formula

$$
\tilde{n}(k ; z)=\frac{1}{\zeta(k ; z)} \int \mathrm{d} w \int_{0}^{\infty} \mathrm{d} \tau \exp \left[-i k\left(w \tau-\epsilon \frac{\tau^{2}}{2}\right)-(z+1) \tau\right] \hat{F}_{\text {in }}(k, w-\epsilon \tau)
$$

with

$$
\zeta(k ; z)=1-\int_{0}^{\infty} \mathrm{d} \tau \exp \left[-(z+1) \tau-\left(i k \epsilon+k^{2}\right) \frac{\tau^{2}}{2}\right]
$$

The insertion of (24) into (23) provides a complete solution for $\tilde{F}(k, w ; z)$ corresponding to a given initial condition.

Formula (24) shows that the time-dependence of the spatial distribution is defined by roots of the function $\zeta(k ; z)$. In order to find the long-time hydrodynamic mode $z_{\text {hy }}(k)$, we have to look for the root of $\zeta(k ; z)$ which approaches 0 when $k \rightarrow 0$. If we assume the asymptotic form

$$
z_{\text {hy }}(k)=c_{1} k+c_{2} k^{2}+o\left(k^{2}\right) \quad \text { when } \quad k \rightarrow 0
$$

we find a unique self-consistent solution to equation $\zeta(k ; z)=0$ of the form

$$
z_{\text {hy }}(k)=-i \epsilon k-\left(1+\epsilon^{2}\right) k^{2}+o\left(k^{2}\right)=-i \epsilon k-D(\epsilon) k^{2}+o\left(k^{2}\right) .
$$

It has the structure of a propagating diffusive mode. It is important to note that the diffusion coefficient $D(\epsilon)$ equals $\left(1+\epsilon^{2}\right)$ in accordance with the Green-Kubo result (21). We thus see that, in the reference system moving with constant velocity $\epsilon$, a classical diffusion process takes place in position space.

It has been argued in the literature that, in general, $z_{\text {hy }}(k)$ is not an analytic function of $k$ at $k=0$ (see e.g. Ref. [9]). Here, that question can be precisely investigated as follows. According to the integral expression (25) of $\zeta(k ; z)$, the hydrodynamic mode is a function of $\xi=i k \epsilon+k^{2}$. By combining differentiations with respect to $\xi$ under the integral sign with integration by parts, we find that $z_{\text {hy }}(\xi)$ satisfies the second order differential equation

$$
\xi \frac{\mathrm{d}^{2} z_{\mathrm{hy}}^{2}}{\mathrm{~d} \xi^{2}}=1+\frac{\mathrm{d} z_{\mathrm{hy}}}{\mathrm{d} \xi}
$$


Then, since $z_{\mathrm{hy}}(0)=0$, we find that $z_{\mathrm{hy}}(\xi)$ can be formally represented by an infinite entire series in $\xi$,

$$
z_{\mathrm{hy}}(\xi)=\sum_{n=1}^{\infty} c_{n} \xi^{n}
$$

with $c_{1}=-1, c_{2}=1$ and

$$
\left|c_{n+1}\right| \geq 2^{n-1} n \text { ! for } n \geq 2 \text {. }
$$

Thus, the radius of convergence of Taylor series (28) is zero, so $\xi=0$ is a singular point of function $z_{\text {hy }}(\xi)$, as well as $k=0$ is a singular point of function $z_{\text {hy }}(k)$. The nature of that singularity can be found by rewriting the root equation defining $z_{\text {hy }}(\xi)$ as the implicit equation

$$
1-\operatorname{Erf}\left(\frac{z_{\mathrm{hy}}+1}{\sqrt{2 \xi}}\right)=\sqrt{\frac{2 \xi}{\pi}} \exp \left(-\frac{\left(z_{\mathrm{hy}}+1\right)^{2}}{2 \xi}\right) .
$$

The introduction of function $\sqrt{\xi}$ requires to define cut-lines ending at points $k=0$ and $k=-i \epsilon$ which are the two roots of equation $\xi(k)=0$. Since the integral in the r.h.s. of expression (25) diverges for $k$ imaginary of the form $k=i q$ with $q>0$ or $q<-\epsilon$, it is natural to define such cut-lines as $[i 0, i \infty[$ and $]-i \infty,-i \epsilon]$. The corresponding choice of determination for $\sqrt{\xi}$ is defined by $\sqrt{\xi\left(k^{+}\right)}=i \sqrt{q \epsilon+q^{2}}$ for $k^{+}=0^{+}+i q$ with $q>0$, where $\sqrt{q \epsilon+q^{2}}$ is the usual real positive square root of the real positive number $\left(q \epsilon+q^{2}\right)$. Notice that, when complex variable $k$ makes a complete tour around point $k=0$ starting from $k^{+}=0^{+}+i q$ on one side of the cut-line and ending at $k^{-}=0^{-}+i q$ on the other side (with vanishing difference $\left.k^{+}-k^{-}\right), \sqrt{\xi(k)}$ changes sign from $\sqrt{\xi^{+}}$to $\sqrt{\xi^{-}}=-\sqrt{\xi^{+}}$with obvious notations. As shown by adding both implicit equations (29) for $k^{+}$and $k^{-}$respectively, $z_{\text {hy }}^{+}$ does not reduce to $z_{\text {hy }}^{-}$. The difference $\left(z_{\text {hy }}^{+}-z_{\text {hy }}^{-}\right)$is of order $\exp (-1 /(2|k| \epsilon))$, so $k=0$ is an essential singularity.

\section{VERY HARD PARTICLES}

Another interesting case is that of the so-called very hard particle model, where the collision frequency is proportional to the kinetic energy of the relative motion of the colliding pair. The corresponding exponent in the collision term of the Boltzmann equation (1) is now $\gamma=2$. This allows us to simplify the resolution of the 
kinetic equation. Owing to this fact, the very hard particle model, similarly to the Maxwell gas, has been studied in numerous works (see e.g. [10]-[11], and references given therein).

Using dimensionless variables (4), we thus write the kinetic equation as

$$
\begin{gathered}
\left(\frac{\partial}{\partial \tau}+w \frac{\partial}{\partial x}+\epsilon \frac{\partial}{\partial w}\right) F(x, w ; \tau)=\int \mathrm{d} u|w-u|^{2}[F(x, u ; \tau) \Phi(w)-F(x, w ; \tau) \Phi(u)] \\
=\left[w^{2} M_{0}(x ; \tau)-2 w M_{1}(x ; \tau)+M_{2}(x ; \tau)\right] \Phi(w)-\left(w^{2}+1\right) F(x, w ; \tau)
\end{gathered}
$$

where the moments $M_{j}(x ; \tau)(j=1,2, \ldots)$ are defined by

$$
M_{j}(x ; \tau)=\int d w w^{j} F(x, w ; \tau) .
$$

The evolution equation of the velocity distribution $G(w ; \tau)$ becomes

$$
\left(\frac{\partial}{\partial \tau}+\epsilon \frac{\partial}{\partial w}\right) G(w ; \tau)=\left[N_{2}(\tau)-2 w N_{1}(\tau)+w^{2} N_{0}\right] \Phi(w)-\left(w^{2}+1\right) G(w ; \tau),
$$

with the integrated moments

$$
N_{j}(\tau)=\int \mathrm{d} x M_{j}(x ; \tau), \quad j=0,1,2 .
$$

Notice that the integrated zeroth moment does not depend on time since the evolution conserves the initial normalization condition

$$
N_{0}(\tau)=\int \mathrm{d} w \int \mathrm{d} x F(x, w ; \tau)=N_{0}
$$

Hence, when $F(x, w ; \tau)$ is a normalized probability density $N_{0}(\tau)=N_{0}=1$.

The simplification related to the choice $\gamma=2$, and more generally when $\gamma$ is an even integer, concerns the collision term in the general kinetic equation (1) which can be expressed in such cases in terms of a finite number of moments of the distribution function. The resolution of that equation becomes then straightforward within standard methods (see Appendix A).

\section{A. Laplace transform of the velocity distribution}

The expression for the Laplace transform of the normalized velocity distribution follows directly from the general formula (A4) derived in Appendix A by putting 
$k=0$, and choosing $\tilde{M}_{0}(0, z)=\tilde{N}_{0}(z)=1 / z$. Within definition

$$
S(w ; z)=(z+1) w+\frac{w^{3}}{3}
$$

for the function $S(k, w ; z)$ evaluated at $k=0$ (see definition (A2)), we find

$$
\begin{aligned}
& \epsilon \tilde{G}(w ; z)=\frac{\epsilon}{z} \Phi(w)+\int_{-\infty}^{w} \mathrm{~d} u \exp \{ {[S(u ; z)-S(w ; z)] / \epsilon\}\left\{G_{\text {in }}(u)\right.} \\
&\left.+\left[\tilde{N}_{2}(z)-2 u \tilde{N}_{1}(z)+\frac{(\epsilon u-z-1)}{z}\right] \Phi(u)\right\} .
\end{aligned}
$$

The two functions $\tilde{N}_{1}(z)$ and $\tilde{N}_{2}(z)$ satisfy the system of equations

$$
\begin{aligned}
0 & =A_{00}^{(\mathrm{in})}(0 ; z)+\left[\tilde{N}_{2}(z)-(z+1) / z\right] A_{00}(0 ; z)+\left[\epsilon / z-2 \tilde{N}_{1}(z)\right] A_{01}(0 ; z) \\
\epsilon \tilde{N}_{1}(z) & =A_{10}^{(\text {in })}(0 ; z)+\left[\tilde{N}_{2}(z)-(z+1) / z\right] A_{10}(0 ; z)+\left[\epsilon / z-2 \tilde{N}_{1}(z)\right] A_{11}(0 ; z)(36)
\end{aligned}
$$

which is identical to (A8) taken at $k=0$, while

$$
A_{j l}(0 ; z)=\int \mathrm{d} w \int_{-\infty}^{w} \mathrm{~d} u \exp \{[S(u ; z)-S(w ; z)] / \epsilon\} w^{j} u^{l} \Phi(u) .
$$

Analogous formula holds for $A_{j l}^{(\mathrm{in})}(0 ; z)$ with the Maxwell distribution $\Phi(u)$ replaced by the initial condition $G(u ; 0)=G_{\text {in }}(u)$. Once system (36) has been solved, the insertion of the resulting expressions for $\tilde{N}_{1}(z)$ and $\tilde{N}_{2}(z)$ into formula (35) yields eventually an explicit solution of the kinetic equation for the velocity distribution

$$
\begin{aligned}
\tilde{G}(w ; z)=\frac{\Phi(w)}{z}+\frac{1}{\epsilon} \int_{-\infty}^{w} \mathrm{~d} u \exp \{[S(u ; z)-S(w ; z)] / \epsilon\} & \\
& \times\left\{G_{\text {in }}(u)+\left[A_{\epsilon}(z) u-B_{\epsilon}(z)\right] \Phi(u)\right\} .
\end{aligned}
$$

With the shorthand notations $A_{j l}(z)=A_{j l}(0 ; z)$ and $A_{j l}^{(\mathrm{in})}(z)=A_{j l}^{(\mathrm{in})}(0 ; z)$, the formulae for coefficients $A_{\epsilon}(z)$ and $B_{\epsilon}(z)$ read

$$
A_{\epsilon}(z)=\frac{1}{\Delta(z)}\left[\frac{\epsilon^{2}}{z} A_{00}(z)-2 A_{00}(z) A_{10}^{(\mathrm{in})}(z)+2 A_{10}(z) A_{00}^{(\mathrm{in})}(z)\right]
$$

and

$$
B_{\epsilon}(z)=\frac{1}{\Delta(z)}\left[\frac{\epsilon^{2}}{z} A_{01}(z)+\epsilon A_{00}^{(\mathrm{in})}(z)+2 A_{11}(z) A_{00}^{(\mathrm{in})}(z)-2 A_{01}(z) A_{10}^{(\mathrm{in})}(z)\right],
$$

where $\Delta(z)$, in accordance with the definition given in (A10), is

$$
\Delta(z)=\epsilon A_{00}(z)+2\left(A_{00}(z) A_{11}(z)-A_{10}(z) A_{01}(z)\right)
$$




\section{B. Stationary solution}

At large times, $\tau \rightarrow \infty$, we expect the velocity distribution to reach some stationary state $G_{\mathrm{st}}(w)=G(w ; \infty)$. This can be easily checked by investigating the behaviour of $\tilde{G}(w ; z)$ in the neighbourhood of $z=0$ at fixed velocity $w$.

All integrals over $u$ in formula (38) do converge for any complex value of $z$. Moreover, all their derivatives with respect to $z$ are also well defined, as shown by differentiation under the integral sign. Thus, such integrals are entire functions of $z$. The sole quantities in expression (38) which become singular at $z=0$ are the coefficients $A_{\epsilon}(z)$ and $B_{\epsilon}(z)$, and obviously the term $\Phi(w) / z$. In fact, both $A_{\epsilon}(z)$ and $B_{\epsilon}(z)$ exhibit simple poles at $z=0$. Hence, the stationary solution of the kinetic equation (32) does emerge when $\tau \rightarrow \infty$, and it is given by the residue of the simple pole of $\tilde{G}(w ; z)$ at $z=0$, namely

$$
G_{\mathrm{st}}(w)=\Phi(w)+\frac{\epsilon}{\Delta(0)} \int_{-\infty}^{w} \mathrm{~d} u \exp \left[\frac{S(u ; 0)-S(w ; 0)}{\epsilon}\right]\left[A_{00}(0) u-A_{01}(0)\right] \Phi(u) .
$$

In that expression, $A_{i j}(0)$ and $\Delta(0)$ are the non-zero values at $z=0$ of the analytic functions $A_{i j}(z)=A_{i j}(0 ; z)$ and $\Delta(z)=\Delta(0 ; z)$. Formula (42) does not depend on initial condition $G_{\text {in }}$. All initial conditions evolve towards the same unique stationary distribution (42). It can be checked that the direct resolution of the static version of kinetic equation (32) obtained by setting $\partial G / \partial \tau=0$ does provide formula (42).

Since the external field accelerates the particle, the stationary solution is asymmetric with respect to the reflection $w \rightarrow-w$, and positive velocities are favoured. This leads to a finite current

$$
\langle w\rangle_{\mathrm{st}}=\int \mathrm{d} w w G_{\mathrm{st}}(w)=\frac{\epsilon}{\Delta(0)}\left[A_{00}(0) A_{11}(0)-A_{01}(0) A_{10}(0)\right] .
$$

The asymptotic expansion at large velocities of $G_{\mathrm{st}}(w)$, inferred from formula (42), reads

$$
G_{\mathrm{st}}(w)=\frac{1}{\sqrt{2 \pi}} e^{-w^{2} / 2}\left[1+\frac{\epsilon^{2} A_{00}(0)}{\Delta(0) w}+O\left(\frac{1}{w^{2}}\right)\right] \quad \text { when } \quad|w| \rightarrow \infty .
$$

Therefore, the external field does not influence the leading large-velocity behaviour of $G_{\mathrm{st}}(w)$, which is identical to that of the thermal bath. Its effects only arise in the 


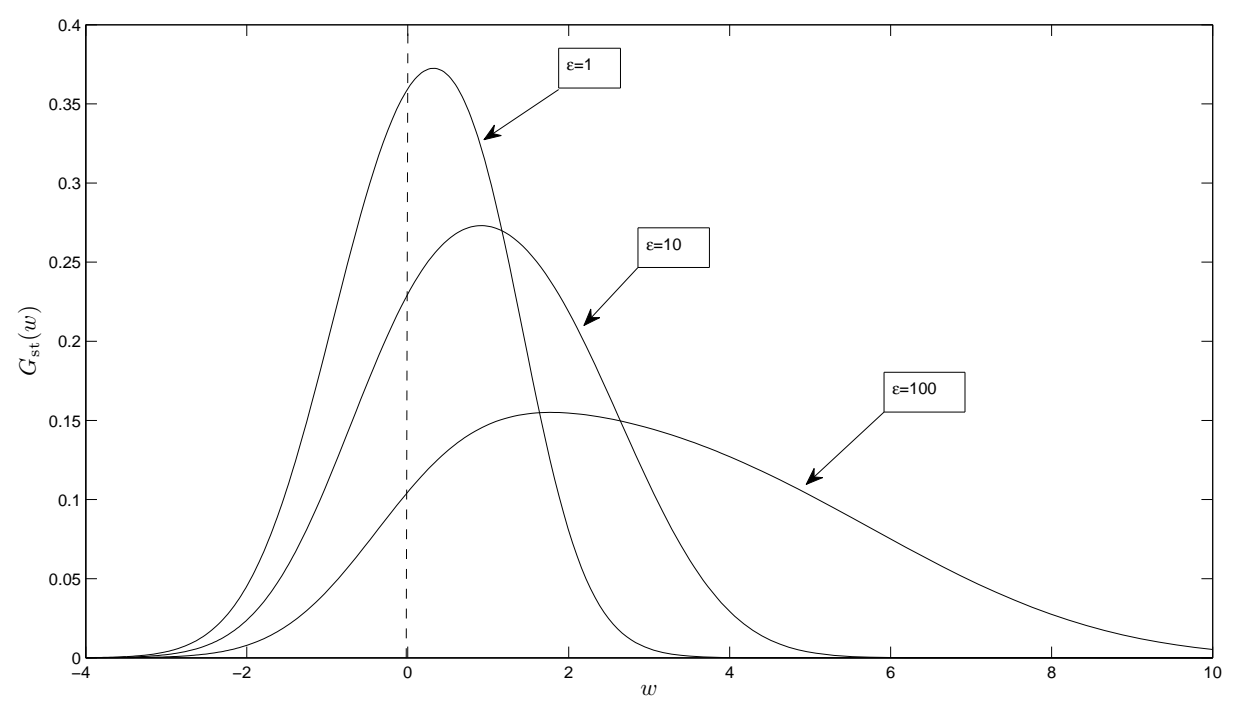

FIG. 2: Stationary velocity distribution $G_{\mathrm{st}}(w)$ for three values of $\epsilon$.

first correction to the leading behaviour which is smaller by a factor of order $1 / w$. The stationary distribution is drawn in Fig. 2 for several increasing field strengths, $\epsilon=1, \epsilon=10$ and $\epsilon=100$.

Let us study now the limit $\epsilon \rightarrow 0$ which corresponds to a weak external field. The main contributions to the integrals over $u$ in (38) arise from the region close to $w$. That observation motivates the use of a new integration variable $y=(w-u) / \epsilon$. The Taylor expansions of the resulting integrands in powers of $\epsilon$ generate then entire series in $\epsilon$, the first terms of which read

$$
\int_{-\infty}^{w} \mathrm{~d} u u \Phi(u) \exp \left[\frac{S(u ; 0)-S(w ; 0)}{\epsilon}\right]=\epsilon \frac{w \Phi(w)}{1+w^{2}}+O\left(\epsilon^{2}\right)
$$

and

$$
\int_{-\infty}^{w} \mathrm{~d} u \Phi(u) \exp \left[\frac{S(u, 0)-S(w, 0)}{\epsilon}\right]=\epsilon \frac{\Phi(w)}{1+w^{2}}+O\left(\epsilon^{2}\right) .
$$

Consequently, also functions $A_{i j}(0)$ and $\Delta(0)$ can be represented by power series in $\epsilon$ as they are obtained by calculating appropriate moments of expansions (45) and (46) over the velocity space. The corresponding small- $\epsilon$ expansion of the stationary velocity distribution reads

$$
G_{\mathrm{st}}(w)=\Phi(w)+\epsilon\left[\frac{b w}{1+w^{2}}\right] \Phi(w)+O\left(\epsilon^{2}\right),
$$


where

$$
b=\left[1+2 \int \mathrm{d} w \frac{w^{2}}{1+w^{2}} \Phi(w)\right]^{-1} .
$$

Of course, at $\epsilon=0, G_{\mathrm{st}}(w)$ reduces to the Maxwell distribution. The first correction is of order $\epsilon$, as expected from linear response theory. The corresponding current (42) reduces to

$$
\langle w\rangle_{\mathrm{st}}=\sigma \epsilon+O\left(\epsilon^{2}\right)
$$

where the conductivity $\sigma$ is given by

$$
\sigma=\frac{1}{2}(1-b)
$$

It will be shown in the sequel that $\sigma=D_{0}=D(\epsilon=0)$, where $D(\epsilon)$ is the diffusion coefficient given by the Green-Kubo formula.

Consider now the strong field limit $\epsilon \rightarrow \infty$. The corresponding behaviours of $A_{i j}(0)$ and $\Delta(0)$ are derived from the integral representations obtained in Appendix $\mathbf{Q}$. We then find at fixed $w$

$$
G_{\mathrm{st}}(w)=\frac{\epsilon^{-1 / 3}}{\int_{0}^{\infty} \mathrm{d} y \exp \left(-y^{3} / 3\right)} \int_{-\infty}^{w} \mathrm{~d} u \Phi(u) \exp \left[\frac{S(u, 0)-S(w, 0)}{\epsilon}\right]+O\left(\epsilon^{-2 / 3}\right)
$$

For $w$ of order 1 , the dominant term in the large- $\epsilon$ expansion of the integral in (50) reduces to

$$
\int_{-\infty}^{w} \mathrm{~d} u \Phi(u)=\frac{1}{2}\left(1+\operatorname{Erf}\left(\frac{w}{\sqrt{2}}\right)\right)
$$

and thus varies from 0 to 1 around the origin $w=0$. For larger values of the velocity, $w \sim \epsilon^{1 / 3}$, that integral behaves as $\exp \left(-w^{3} /(3 \epsilon)\right.$. The next term in the expansion (50) remains of order $\epsilon^{-2 / 3}$. Thus, when $\epsilon \rightarrow \infty$ at fixed $\epsilon^{-1 / 3} w$ the stationary solution is given by

$$
G_{\mathrm{st}}(w) \sim \theta(w) \frac{\epsilon^{-1 / 3}}{\int_{0}^{\infty} \mathrm{d} y \exp \left(-y^{3} / 3\right)} \exp \left[-\left(\epsilon^{-1 / 3} w\right)^{3} / 3\right],
$$

where $\theta$ is the Heaviside step function. The whole distribution is shifted toward high velocities $w \sim \epsilon^{1 / 3}$, so that the resulting current (43) is of the same order of magnitude, i.e.

$$
\langle w\rangle_{\mathrm{st}} \sim \frac{3^{1 / 3} \Gamma(2 / 3)}{\Gamma(1 / 3)} \epsilon^{1 / 3} \text { when } \epsilon \rightarrow \infty
$$

where $\Gamma$ is the Euler Gamma function. That behavior can be recovered within the following simple interpretation. At strong fields, the average velocity of the 


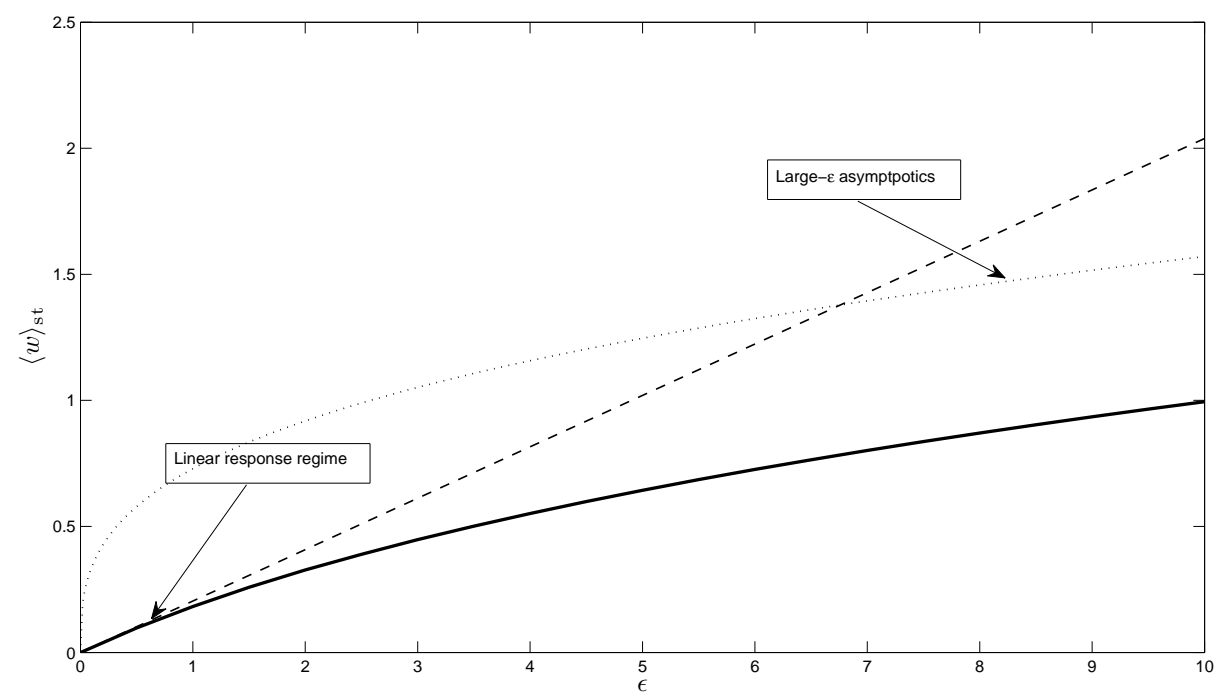

FIG. 3: Average current $\langle w\rangle_{\text {st }}$ as a function of $\epsilon$. The dashed line represents the linear Kubo term in the small- $\epsilon$ expansion (48) with conductivity $\sigma \simeq 0.2039$. The dotted line describes asymptotic formula (52) with $3^{1 / 3} \Gamma(2 / 3) / \Gamma(1 / 3) \simeq 0.7290$ valid in the limit $\epsilon \rightarrow \infty$

particle becomes large compared to the thermal velocity of scatterers. Since at each collision the particle exchanges its velocity with a thermalized scatterer, the variation of particle velocity between two successive collisions is of the order of $\langle v\rangle_{\mathrm{st}}$. On the other hand, in the stationary state the same velocity variation is due to the acceleration $a$ coming from the external field, so it is of the order $a \tau_{\text {coll }}$ where $\tau_{\text {coll }}$ is the mean time between two successive collisions. This time can be reasonably estimated as the inverse collision frequency for a relative velocity $|v-c|$ of order $\langle v\rangle_{\mathrm{st}}$. The consistency of those estimations requires the relation

$$
\langle v\rangle_{\mathrm{st}} \sim a \frac{v_{\mathrm{int}}}{\rho\langle v\rangle_{\mathrm{st}}^{2}}
$$

which indeed implies the $\epsilon^{1 / 3}$-behaviour (52) of the average velocity in dimensionless units. Contrary to the Maxwell case where the current remains linear in the applied field, here the current deviates from its linear-response form when the field increases : it grows more slowly because collisions are more efficient in dissipating the energy input of the field. In Fig. 3, we plot $\langle w\rangle_{\text {st }}$ as a function of $\epsilon$. 


\section{Relaxation towards the stationary solution}

Let us study now the relaxation of the velocity distribution $G(w ; \tau)$ towards the stationary solution $G_{\mathrm{st}}(w)$. The decay of $\left[G(w, \tau)-G_{\mathrm{st}}(w)\right]$ when $\tau \rightarrow \infty$ is controlled by the singularities of $\tilde{G}(w ; z)$ in the complex plane, different from the pole at $z=0$. As already mentioned, all integrals in expression (38) are entire functions of $z$, so the singularities at $z \neq 0$ arise only in the coefficients $A_{\epsilon}(z)$ and $B_{\epsilon}(z)$. Thus, the first important conclusion is that the relaxation is uniform for the whole velocity spectrum.

According to expressions (39) and (40) defining $A_{\epsilon}(z)$ and $B_{\epsilon}(z)$ respectively, the singularities of those coefficients at points $z \neq 0$, correspond to zeros of the function $\Delta(z)$ given by expression (41). Since the analytic functions $A_{i j}(z)$ and $\Delta(z)$ do not depend on initial condition $G_{\text {in }}$. the relaxation is an intrinsic dynamical process, as expected.

After some algebra detailed in Appendix $\mathrm{C}$, we find that $\Delta(z)$ reduces to the Laplace transform

$$
\Delta(z)=\epsilon^{2} \int_{0}^{\infty} \mathrm{d} y f_{\epsilon}(y) \exp (-z y)
$$

of the real, positive, and monotonously decreasing function

$$
\epsilon^{2} f_{\epsilon}(y)=\frac{\epsilon^{2}(1+3 y)}{(1+y)(1+2 y)^{1 / 2}} \exp \left(-y-\epsilon^{2} \frac{y^{3}(2+y)}{6(1+2 y)}\right) .
$$

Owing to the fast decay of $f_{\epsilon}(y)$ the integral (54) converges for any $z$, so $\Delta(z)$ is an entire function of $z$. Also, the monotonic decay of $f_{\epsilon}(y)$ and its positivity imply some general properties for the roots of $\Delta(z)$. First of all, $\Delta(z)$ cannot vanish for $\Re(z) \geq 0$. Moreover, as $\Delta(z)$ is strictly positive for $z$ real, the zeros of $\Delta(z)$ appear in complex conjugate pairs, while they are isolated with strictly negative real parts and nonvanishing imaginary parts. Consequently, the long-time relaxation of the velocity distribution is governed by the pair of zeros which is closest to the imaginary axis. Noting them as $z^{ \pm}=-\lambda \pm i \omega$ with $\omega \neq 0$ and $0<\lambda$, we conclude that $G(w ; \tau)$ relaxes towards $G_{\text {st }}(w)$ via exponentially damped oscillations

$$
G(w ; \tau)-G_{\mathrm{st}}(w) \sim C(w) \cos [\omega \tau+\eta(w)] \exp (-\lambda \tau), \text { when } \tau \rightarrow \infty
$$


where $C(w)$ and $\eta(w)$ are an amplitude and a phase respectively. It should be noticed that both functions $C(w)$ and $\eta(w)$ depend on initial conditions.

At a given value of $\epsilon$, the zeros $z^{ \pm}$are found by solving numerically the equation $\Delta\left(z^{ \pm}\right)=0$. In the weak- or strong-field limits, we can derive asymptotic formulae for such zeros as follows. First, as indicated by numerically computing $z^{ \pm}$for small values of $\epsilon, z^{ \pm}$collapse to $z_{0}=-1$ when $\epsilon \rightarrow 0$. The corresponding asymptotical behaviour can be derived by noting that, for $z$ close to $z_{0}$, the leading contributions to $\Delta(z)$ in integral (54) arise from large values of $y$. Then, we set $y=\xi / \epsilon^{2 / 3}$ and $z=-1+s \epsilon^{2 / 3}$, which provide

$$
\Delta\left(-1+s \epsilon^{2 / 3}\right) \sim \frac{3 \epsilon^{5 / 3}}{\sqrt{2}} \int_{0}^{\infty} \mathrm{d} \xi \xi^{-1 / 2} \exp \left(-s \xi-\xi^{3} / 12\right)
$$

when $\epsilon \rightarrow 0$ at fixed $s$. By numerical methods, we find the pair of complex conjugated zeros $s_{0}^{ \pm}$of integral

$$
\int_{0}^{\infty} \mathrm{d} \xi \xi^{-1 / 2} \exp \left(-s \xi-\xi^{3} / 12\right)
$$

which are the closest to the imaginary axis. Therefore, when $\epsilon \rightarrow 0$, damping factor $\lambda(\epsilon)$ goes to 1 according to

$$
\lambda(\epsilon)=1-\Re\left(s_{0}^{ \pm}\right) \epsilon^{2 / 3}+o\left(\epsilon^{2 / 3}\right)
$$

with $\Re\left(s_{0}^{ \pm}\right) \simeq-1.169$, while frequency $\omega(\epsilon)$ vanishes as $\Im\left(s_{0}^{+}\right) \epsilon^{2 / 3}$ with $\Im\left(s_{0}^{+}\right) \simeq$ 2.026. Notice that for fixed $z$, not located on the real half-axis ] $-\infty,-1], \Delta(z)$ behaves as

$$
\Delta(z) \sim \epsilon^{2} \Delta_{0}(z)
$$

when $\epsilon \rightarrow 0$, with

$$
\begin{aligned}
\Delta_{0}(z)=\sqrt{\frac{\pi}{2(z+1)}} e^{(z+1) / 2}[1-\operatorname{Erf}(\sqrt{(z+1) / 2})] \\
\times\left[3-\sqrt{2 \pi(z+1)} e^{(z+1) / 2}(1-\operatorname{Erf}(\sqrt{(z+1) / 2}))\right] .
\end{aligned}
$$

Here, $\sqrt{(z+1) / 2}$ is defined as the usual real positive square root $\sqrt{(x+1) / 2}$ for real $z=x$ belonging to the half axis $x>-1$, while the complementary half-axis $z=x \leq-1$ is a cut-line ending at the branching point $z=-1$. That point is the singular point of $1 / \Delta_{0}(z)$ closest to the imaginary axis, as strongly suggested by a 
numerical search of the zeros of $\Delta_{0}(z)$. Therefore, both $\lambda(\epsilon)$ and $\omega(\epsilon)$ are continuous functions of $\epsilon$ at $\epsilon=0$ with $\lambda(0)=1$ and $\omega(0)=0$. At $\epsilon=0$, the exponentially damped oscillating decay (56) becomes an exponentially damped monotonic decay multiplied by power-law $t^{-3 / 2}$. That power-law arises from the presence of a singular term of order $\sqrt{(z+1) / 2}$ in the expansion of $\tilde{G}(w ; z)$ around the branching point $z=-1$.

When $\epsilon \rightarrow \infty$, the zeros of $\Delta(z)$ are obtained by simultaneously changing $y$ to $\xi / \epsilon^{2 / 3}$ in the integral (54) and by rescaling $z$ as $\epsilon^{2 / 3} s$. This provides

$$
\Delta\left(\epsilon^{2 / 3} s\right) \sim \epsilon^{4 / 3} \Delta_{\infty}(s) \text { when } \epsilon \rightarrow \infty \text { at fixed } s,
$$

with

$$
\Delta_{\infty}(s)=\int_{0}^{\infty} \mathrm{d} \xi \exp \left(-s \xi-\xi^{3} / 3\right)
$$

Therefore, when $\epsilon \rightarrow \infty, z^{ \pm}$behave as $z^{ \pm} \sim \epsilon^{2 / 3} s_{\infty}^{ \pm}$, where $s_{\infty}^{ \pm}$are the zeros of $\Delta_{\infty}(s)$ closest to the imaginary axis. The corresponding large- $\epsilon$ asymptotical behaviour of the damping factor $\lambda(\epsilon)$ is

$$
\lambda(\epsilon)=-\Re\left(s_{\infty}^{ \pm}\right) \epsilon^{2 / 3}+o\left(\epsilon^{2 / 3}\right)
$$

with $\Re\left(s_{\infty}^{ \pm}\right) \simeq-2.726$, while frequency $\omega(\epsilon)$ diverges as $\Im\left(s_{\infty}^{+}\right) \epsilon^{2 / 3}$ with $\Im\left(s_{\infty}^{+}\right) \simeq$ 6.260. Notice that the relaxation time $\lambda^{-1}(\epsilon)$ goes to zero as $\epsilon^{-2 / 3}$, like the average time between collisions $\tau_{\text {coll }} \sim\langle v\rangle_{\text {st }} / a$ used in our simple heuristic derivation of the

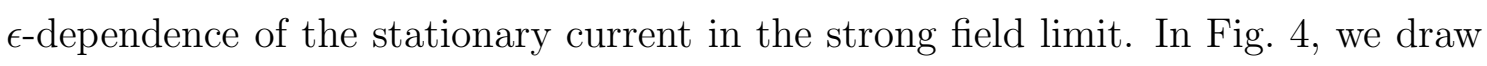
the damping factor $\lambda(\epsilon)$ as a function of $\epsilon$.

\section{Relaxation of density in position space}

In Appendix $\mathrm{A}$ we derive an explicit formula for the zeroth moment $\tilde{M}_{0}(k ; z)$ of the distribution $\tilde{F}(k, w ; z)$ which contains all information on the evolution of the spatial density of the propagating particle. The formula (A9) clearly reveals the presence of a hydrodynamic pole in $\tilde{M}_{0}(k ; z)$, namely the root of equation

$$
z+\left(k^{2}+i \epsilon k\right) U(k ; z)=0
$$




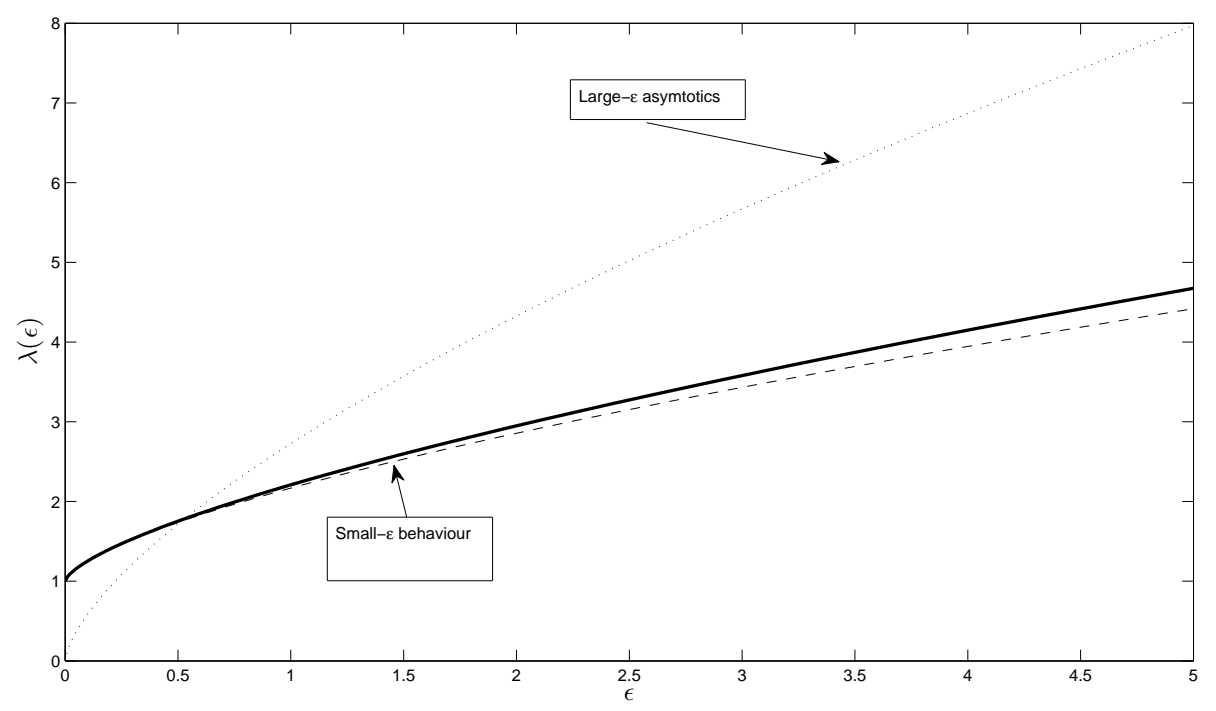

FIG. 4: Damping factor $\lambda(\epsilon)$ as a function of $\epsilon$. The dashed and dotted lines represent the asymptotical behaviours (58) and (63) at small and large $\epsilon$ respectively.

where

$$
U(k ; z)=\frac{A_{11}(k ; z) A_{00}(k ; z)-A_{10}(k ; z) A_{01}(k ; z)}{\epsilon A_{00}(k ; z)+2\left[A_{11}(k ; z) A_{00}(k ; z)-A_{10}(k ; z) A_{01}(k ; z)\right]} .
$$

If we consider the small- $k$ limit and if we assume the asymptotic form

$$
z_{\text {hy }}(k)=-i c k-D(\epsilon) k^{2}+0\left(k^{2}\right)
$$

for the hydrodynamic root, we find immediately from equation (64) the formula

$$
c=\epsilon U(0 ; 0) \text {. }
$$

This shows that the mode propagates with the average stationary velocity $\langle w\rangle_{\mathrm{st}}=$ $\epsilon U(0 ; 0)$ derived in expression (43).

In order to infer the formula for the diffusion coefficient $D(\epsilon)$, it is necessary to calculate the term linear in variable $k$ in the expansion of function $U(k ; z)$ at $z=-i c k$. Indeed, equation (64) implies the equality

$$
D(\epsilon)=U(0 ; 0)+\left.i \epsilon \frac{\mathrm{d}}{\mathrm{d} k} U(k ;-i c k)\right|_{k=0} .
$$

Taking into account the structure (68) of $U(k ; z)$ we find the formula

$$
D(\epsilon)=\frac{\langle w\rangle_{\mathrm{st}}}{\epsilon}+\frac{A_{00}\left[A_{11}^{\prime} A_{00}-A_{01}^{\prime} A_{10}\right]+A_{01}\left[A_{00}^{\prime} A_{10}-A_{10}^{\prime} A_{00}\right]}{\Delta^{2}}
$$


where all $A_{j l}$ and $\Delta$ are taken at $k=z=0$, and where

$$
A_{j l}^{\prime}=\left.i \epsilon \frac{\mathrm{d}}{\mathrm{d} k} A_{j l}(k ;-i c k)\right|_{k=0} .
$$

A particularly useful representation of the derivative appearing in expression (70) can be deduced from formulae (A2) and (A6) defining functions $A_{j l}(k ; z)$. An integration by parts yields

$$
A_{j l}^{\prime}=\int \mathrm{d} w \int_{-\infty}^{w} \mathrm{~d} u(u-c) \int_{-\infty}^{u} \mathrm{~d} v w^{j} v^{l} \exp \{[S(0, v ; 0)-S(0, w ; 0)] / \epsilon\} \Phi(v) .
$$

It is quite remarkable that equation (71) allows us to establish a relation between the quantities $A_{j l}^{\prime}$ and the stationary velocity distribution $G_{\mathrm{st}}(w)$. Indeed, using equation (42), we readily obtain the equalities

$$
\begin{aligned}
\int \mathrm{d} w \int_{-\infty}^{w} \mathrm{~d} u \exp \{[S(0, v ;)-S(0, w ; 0)] / \epsilon\}(u-c) G_{\mathrm{st}}(u) & \\
& =A_{01}-c A_{00}+\frac{1}{\Delta}\left[A_{00} A_{01}^{\prime}-A_{01} A_{00}^{\prime}\right] \equiv J_{01}
\end{aligned}
$$

and

$$
\begin{aligned}
\int \mathrm{d} w \int_{-\infty}^{w} \mathrm{~d} u \exp \{[S(0, v ;)- & S(0, w ; 0)] / \epsilon\} w(u-c) G_{\mathrm{st}}(u) \\
& =A_{11}-c A_{10}+\frac{1}{\Delta}\left[A_{00} A_{11}^{\prime}-A_{01} A_{10}^{\prime}\right] \equiv J_{11} .
\end{aligned}
$$

Then, we find that the linear combination $\left(A_{00} J_{11}-A_{10} J_{01}\right)$ of integrals $J_{11}$ and $J_{01}$ reduces to

$$
A_{11} A_{00}-A_{10} A_{01}+\frac{1}{\Delta}\left\{A_{00}\left[A_{00} A_{11}^{\prime}-A_{01} A_{10}^{\prime}\right]-A_{10}\left[A_{00} A_{01}^{\prime}-A_{01} A_{00}^{\prime}\right]\right\} .
$$

The comparison of that expression with equation (69) leads to the compact final result

$$
D(\epsilon)=\frac{A_{00} J_{11}-A_{10} J_{01}}{\Delta}
$$

The above formula involves, via coefficients $J_{11}$ and $J_{01}$, averages over the stationary velocity distribution. In fact, we show in Appendix B that expression (75) follows by extending, to the present out-of-equilibrium stationary state, the familiar GreenKubo relation between the diffusion coefficient and the velocity fluctuations. That important fact is one of the main observations of the present study. 
When $\epsilon \rightarrow 0$, the behaviour of $D(\epsilon)$ is easily infered by inserting the small- $\epsilon$ expansion (47) of the stationary velocity distribution $G_{\mathrm{st}}(w)$ into formula (75). We find that $D(\epsilon)$ goes to conductivity $\sigma(49)$ as quoted above, with a negative $\epsilon^{2}$ correction. When $\epsilon \rightarrow \infty$, we can use the large- $\epsilon$ form (51) of $G_{\mathrm{st}}(w)$ for evaluating coefficients $J_{11}$ and $J_{01}$. Using also the corresponding behaviours of coefficients $A_{00}$ and $A_{10}$, we eventually obtain that $D(\epsilon)$ goes to the finite value

$$
D_{\infty}=\frac{\Gamma^{3}(1 / 3)-9 \Gamma(1 / 3) \Gamma(2 / 3)+6 \Gamma^{3}(2 / 3)}{2 \Gamma^{3}(1 / 3)} \simeq 0.0384 .
$$

The external field dependence of the diffusion coefficient $D(\epsilon)$ is shown in Fig. 5 .

The expansion (66) of $z_{\text {hy }}(k)$ can be pursued beyond the $k^{2}$-diffusion term, by expanding function $U(k ; z)$ in double entire series with respect to $z$ and $k$. According to the integral expression of functions $A_{j l}(k ; z)$ derived in Appendix $\mathrm{C}$, all coefficients of those double series are finite. This implies that the hydrodynamic root $z_{\text {hy }}(k)$ of equation (64) can be formally represented by an entire series in $k$, namely

$$
z_{\mathrm{hy}}(k)=\sum_{n=1}^{\infty} \alpha_{n} k^{n},
$$

with $\alpha_{1}=-i c$ and $\alpha_{2}=-D(\epsilon)$. Coefficient $\alpha_{n}(n \geq 3)$ can be straightforwardly computed once lowest-order coefficients $\alpha_{p}$ with $1 \leq p \leq n-1$ have been determined. As shown by that calculation, all coefficients are obviously finite. Therefore, and similarly to what happens in the Maxwell case, only positive integer powers of $k$ appear in the small- $k$ expansion of $z_{\text {hy }}(k)$. Now, we are not able to determine the radius of convergence of that expansion, so we cannot conclude about the analyticity of function $z_{\text {hy }}(k)$. However, we notice that, contrarily to the Maxwell case, the integrals defining $A_{j l}(k ; z)$ remain well-defined for any complex value of $k$, as soon as $\epsilon \neq 0$ (see Appendic Q). This suggests that $z_{\mathrm{hy}}(k)$ might be an analytic function of $k$ at $k=0$, except for $\epsilon=0$, in which case $k=0$ should be a singular point.

\section{CONCLUDING COMMENTS}

The idea of this work was to perform a detailed study of the approach to an outof-equilibrium stationary state, by considering systems for which analytic solutions 


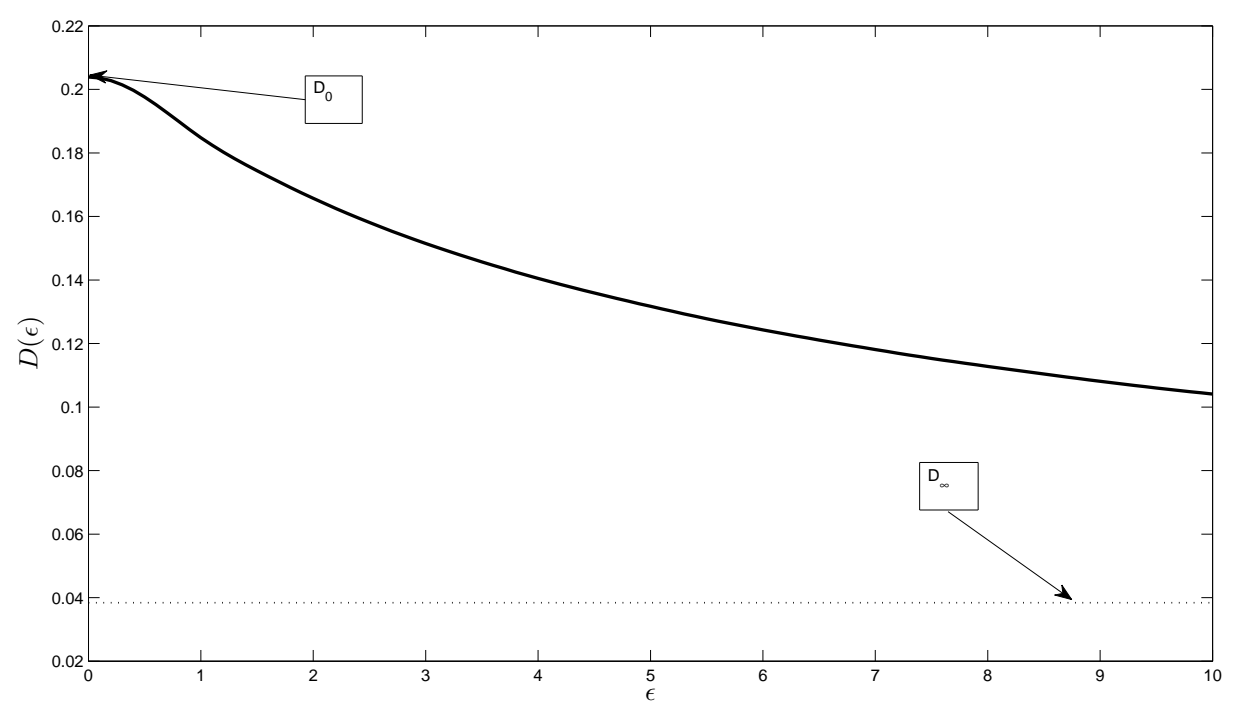

FIG. 5: Diffusion coefficient $D(\epsilon)$ as a function of $\epsilon$. The dotted line represents the constant asymptotic value $D_{\infty}$.

can be derived. To this end we solved, within Boltzmann's kinetic theory, the one-dimensional initial value problem for the distribution of a particle accelerated by a constant external field and suffering elastic collisions with thermalized bath particles. Our exact results for the Maxwell model and for the very hard particle model support the general picture mentioned in the Introduction:

- a uniform exponentially fast relaxation of the velocity distribution

- diffusive spreading in space in the reference system moving with stationary flow

- equality between the diffusion coefficient appearing in the hydrodynamic mode and the one given by the generalized Green-Kubo formula

Although both models display the same phenomena listed above, the variations of the respective quantities of interest with respect to $\epsilon$ are different. First we notice that, as far as deformations of the equilibrium Maxwell distribution are concerned, the external field is much less efficient for very hard particles. This is well illustrated by comparing figures 1 and 2 : for the Maxwell system, a significative deformation of $\Phi$ is found for $\epsilon=5$, while for the very-hard particle model a similar deformation 
is observed for $\epsilon=100$. This can be easily interpreted as follows. The collision frequency for very hard particles becomes much larger than its Maxwell gas counterpart when the external field increases, so it costs more energy to maintain a stationary distribution far from the equilibrium one. That mechanism also explains various related observations. For instance, the large-velocity behaviour of $G_{\mathrm{st}}(w)$ is identical to the equilibrium Gaussian for very hard particles, while it takes an exponential form in the Maxwell gas. Also, the average current $\langle w\rangle_{\mathrm{st}}$ increases more slowly when $\epsilon \rightarrow \infty$ for very hard particles, and the corresponding relaxation time $\lambda^{-1}(\epsilon)$ vanishes instead of remaining constant for the Maxwell gas.

Among the above phenomena, the emergence of a symmetric diffusion process in the moving reference frame is quite remarkable. In such a frame, there is some kind of cancellation between the action of the external field and the effects of collisions induced by the counterflow of bath particles with velocity $u_{\mathrm{bath}}^{*}=-\langle v\rangle_{\mathrm{st}}$. The corresponding diffusion coefficient $D(\epsilon)$ increases whith $\epsilon$ for the Maxwell gas (case $\gamma=0$ ), while it decreases and saturates to a finite value for very hard particles (case $\gamma=2$ ). Therefore, beyond the previous cancellation, it seems that the large number of collisions for $\gamma=2$ shrink equilibrium fluctuations. On the contrary, for $\gamma=0$, since $D(\epsilon)$ diverges when $\epsilon \rightarrow \infty$, the residual effect of collisions in the reference frame seems to vanish and particles tend to behave as if they were free.

We expect that the same qualitative picture should be valid in the hard rod case which corresponds to the intermediate value $\gamma=1$ of the exponent $\gamma$ in equation (11). The quantitative behaviours should interpolate between those described for $\gamma=0$ and $\gamma=2$. For instance, the stationary distribution $G_{\mathrm{st}}(w)$ computed in Ref. [1] displays a large-velocity asymptotic behaviour which is indeed intermediary between those derived here for $\gamma=0$ and $\gamma=2$. Also, the average current $\langle v\rangle_{\text {st }}$ is of order $\epsilon^{1 / 2}$ for $\epsilon$ large, which lies between the $\epsilon$ - and $\epsilon^{1 / 3}$-behaviours found for $\gamma=0$ and $\gamma=2$ respectively. Notice that the $\epsilon^{1 / 3}$-behaviour for $\gamma=2$ can be retrieved within a selfconsistent argument, which uses in an essential way the existence of the velocity scale related to the particle-particle interaction. Whereas the thermal velocity scale becomes irrelevant when $\epsilon \rightarrow \infty$, the interaction scale remains important. In the case of hard rods such an interaction scale does not show in the kinetic equation, 
and the unique combination of parameters having the dimension of velocity is $\sqrt{a / \rho}$, which does provide a different strong field behaviour of $\langle v\rangle_{\text {st }}$ with order $\epsilon^{1 / 2}$.

\section{Appendix A: Solution of the kinetic equation for very hard particles}

Applying to equation (30) Fourier and Laplace transformations, we find

$$
\begin{aligned}
\epsilon \frac{\partial}{\partial w} \tilde{F}(k, w ; z)+ & \left(z+1+i k w+w^{2}\right) \tilde{F}(k, w ; z) \\
& =\hat{F}_{\text {in }}(k, w)+\left[\tilde{M}_{2}(k ; z)-2 w \tilde{M}_{1}(k ; z)+w^{2} \tilde{M}_{0}(k ; z)\right] \Phi(w)
\end{aligned}
$$

where $\tilde{M}_{j}(k ; z)$ is the double Fourier-Laplace transform of the $j^{t h}$-moment $M_{j}(x ; \tau)$ defined in expression (31), while $\hat{F}_{\text {in }}(k, w)$ is the spatial Fourier transform of the initial condition $F_{\text {in }}(x, w)=F(x, w ; 0)$. The first order equation (A1) can be rewritten in an integral form with the use of function

$$
S(k, w ; z)=w(z+1)+\frac{1}{3} w^{3}+i k \frac{w^{2}}{2},
$$

namely

$$
\begin{aligned}
\epsilon \tilde{F}(k, w ; z)=\int_{-\infty}^{w} \mathrm{~d} u \exp \{[ & S(k, u ; z)-S(k, w ; z)] / \epsilon\}\left\{\hat{F}_{\text {in }}(k, u)\right. \\
& \left.+\left[\tilde{M}_{2}(k ; z)-2 u \tilde{M}_{1}(k ; z)+u^{2} \tilde{M}_{0}(k ; z)\right] \Phi(u)\right\} .
\end{aligned}
$$

Using then the relation

$$
\left[\epsilon \frac{\partial}{\partial u}+\epsilon u-(z+1)-i k u-u^{2}\right] \exp \{S(k, u ; z) / \epsilon\} \Phi(u)=0
$$

to evaluate the term involving $u^{2}$ in the right hand side of equation (A3), we eventually find the more convenient integral equation

$$
\begin{aligned}
& \epsilon \tilde{F}(k, w ; z)=\epsilon \tilde{M}_{0}(k ; z) \Phi(w)+\int_{-\infty}^{w} \mathrm{~d} u \exp \{[S(k, u ; z)-S(k, w ; z)] / \epsilon\} \\
& \times\left\{\hat{F}_{\text {in }}(k, u)+\left[\tilde{M}_{2}(k ; z)-2 u \tilde{M}_{1}(k ; z)+(\epsilon u-i k u-z-1) \tilde{M}_{0}(k ; z)\right] \Phi(u)\right\} .
\end{aligned}
$$

Equation (A4) has to be considered together with the continuity equation

$$
z \tilde{M}_{0}(k ; z)+i k \tilde{M}_{1}(k ; z)=\hat{M}_{0}(k ; 0) .
$$


In order to determine the unknown functions $\tilde{M}_{j}(k ; z)(j=0,1,2)$, we complete equation (A5) with the zeroth and the first moments of equation (A4). In the resulting system of linear equations, the integrals

$$
A_{j l}(k ; z)=\int \mathrm{d} w \int_{-\infty}^{w} \mathrm{~d} u w^{j} u^{l} \exp \{[S(k, u ; z)-S(k, w ; z)] / \epsilon\} \Phi(u)
$$

and

$$
A_{j l}^{(\mathrm{in})}(k ; z)=\int \mathrm{d} w \int_{-\infty}^{w} \mathrm{~d} u w^{j} u^{l} \exp \{[S(k, u ; z)-S(k, w ; z)] / \epsilon\} \hat{F}_{\mathrm{in}}(k, u)
$$

appear. That system reads

$$
\begin{aligned}
0 & =A_{00}^{(\mathrm{in})}+\left[\tilde{M}_{2}-(z+1) \tilde{M}_{0}\right] A_{00}+\left[(\epsilon-i k) \tilde{M}_{0}-2 \tilde{M}_{1}\right] A_{01} \\
\epsilon \tilde{M}_{1} & =A_{10}^{(\text {in })}+\left[\tilde{M}_{2}-(z+1) \tilde{M}_{0}\right] A_{10}+\left[(\epsilon-i k) \tilde{M}_{0}-2 \tilde{M}_{1}\right] A_{11}
\end{aligned}
$$

The explicit solution for the zeroth moment reads

$$
\tilde{M}_{0}=\frac{\Delta \hat{M}_{0}(k ; 0)-i k\left[A_{10}^{(\mathrm{in})} A_{00}-A_{10} A_{00}^{(\mathrm{in})}\right]}{z \Delta+\left(k^{2}+i \epsilon k\right)\left(A_{11} A_{00}-A_{10} A_{01}\right)}
$$

where

$$
\Delta=\epsilon A_{00}+2\left(A_{11} A_{00}-A_{10} A_{01}\right) .
$$

The formula for the first moment follows directly from the continuity equation (A5), and then $\tilde{M}_{2}$ can be derived directly from (A8). The insertion of the formulae for the first three moments into the relation (A4) yields the complete solution for the distribution $\tilde{F}(k, w ; z)$ for any initial condition.

\section{Appendix B: Evaluation of the diffusion coefficient via Green-Kubo theory}

\section{Velocity autocorrelation function of the Maxwell gas}

In order to evaluate the velocity autocorrelation function, we use the integral representation

$$
\Gamma(\tau)=<\left[w(\tau)-<w>_{\mathrm{st}}\right]\left[w(0)-<w>_{\mathrm{st}}\right]>_{\mathrm{st}}=\int \mathrm{d} w w G(w ; \tau)
$$

where $G(w ; \tau)$ is the solution of kinetic equation (12) corresponding to the initial condition

$$
G_{\mathrm{in}}(w)=\left(w-<w>_{\mathrm{st}}\right) G_{\mathrm{st}}(w)=(w-\epsilon) G_{\mathrm{st}}(w)
$$


Here we find

$$
N_{0}=\int \mathrm{d} w G(w ; \tau)=\int \mathrm{d} w G(w ; 0)=0,
$$

so that equation (12) takes a particularly simple form

$$
G(w ; \tau)=e^{-\tau} G_{\text {in }}(w-\epsilon \tau)=e^{-\tau}(w-\epsilon) G_{\mathrm{st}}(w-\epsilon \tau) .
$$

Using then the explicit form (14) of $G_{\text {st }}$, we obtain

$$
\Gamma(\tau)=\int \mathrm{d} w w e^{-\tau}(w-\epsilon) \int_{0}^{\infty} \mathrm{d} \eta e^{-\eta} \Phi(w-\epsilon \eta)=e^{-\tau}\left(1+\epsilon^{2}\right)
$$

which leads to the simple formula for the diffusion coefficient

$$
D(\epsilon)=\int_{0}^{\infty} \mathrm{d} \tau \Gamma(\tau)=1+\epsilon^{2} .
$$

\section{Velocity autocorrelation function of very hard particles}

Similarly to the Maxwell gas case, in order to determine the autocorrelation function $\Gamma(\tau)$, we first have to solve kinetic equation (32) satisfied by the velocity distribution with the initial condition $G(w ; 0)=G_{\mathrm{in}}(w)=\left(w-<w>_{\mathrm{st}}\right) G_{\mathrm{st}}(w)$, and afterwards we have to evaluate the first moment of that solution

$$
\int \mathrm{d} w w G(w ; \tau)=\Gamma(\tau)
$$

Since norm $N_{0}(\tau)$ vanishes, kinetic equation (32) becomes

$$
\left(\frac{\partial}{\partial \tau}+\epsilon \frac{\partial}{\partial w}\right) G(w ; \tau)=\left[N_{2}(\tau)-2 w N_{1}(\tau)\right] \Phi(w)-\left(w^{2}+1\right) G(w ; \tau)
$$

That equation can be rewritten in Laplace world as

$$
\begin{aligned}
& \epsilon \tilde{G}(w ; z)=\int_{-\infty}^{w} \mathrm{~d} u \exp \{[S(u ; z)-S(w ; z)] / \epsilon\} \\
& \times\left\{\left(u-<w>_{\mathrm{st}}\right) G_{\mathrm{st}}(u)+\left[\tilde{N}_{2}(z)-2 \tilde{N}_{1}(z) u\right] \Phi(u)\right\} .
\end{aligned}
$$

The zeroth and the first moments of equation (B8) provide the system of equations

$$
\begin{aligned}
0 & =J_{01}+\tilde{N}_{2} A_{00}-2 \tilde{N}_{1} A_{01} \\
\epsilon \tilde{N}_{1} & =J_{11}+\tilde{N}_{2} A_{10}-2 \tilde{N}_{1} A_{11}
\end{aligned}
$$


where

$$
J_{01}=\int \mathrm{d} w \int_{-\infty}^{w} d u \exp \{[S(u ; z)-S(w ; z)] / \epsilon\}\left(u-<w>_{\mathrm{st}}\right) G_{\mathrm{st}}(u)
$$

and

$$
J_{11}=\int \mathrm{d} w \int_{-\infty}^{w} d u \exp \{[S(u ; z)-S(w ; z)] / \epsilon\} w\left(u-<w>_{\mathrm{st}}\right) G_{\mathrm{st}}(u) .
$$

The first moment $\tilde{N}_{1}(z)=\tilde{\Gamma}(z)$ is found to be

$$
\tilde{\Gamma}(z)=\frac{A_{00}(z) J_{11}(z)-A_{10}(z) J_{01}(z)}{\epsilon A_{00}(z)+2\left[A_{00}(z) A_{11}(z)-A_{10}(z) A_{01}(z)\right]},
$$

where the shorthand notation $A_{j l}(z) \equiv A_{j l}(0 ; z)$ has been used. The value of $\tilde{\Gamma}(z)$ at $z=0$ yields the diffusion coefficient $D(\epsilon)$. We find here the same formula as that derived from the analysis of the hydrodynamic pole (see expression (75)).

\section{Appendix C: Useful integral expressions for functions arising in the case of} very hard particles

In the integral representation (A6) of function $A_{j l}(k ; z)$, it is useful to make the variable change $u=w-\epsilon y$. This leads to a double integral of the form

$$
\int \mathrm{d} w \int_{0}^{\infty} \mathrm{d} y I_{j l}(w, y) \text {. }
$$

Thanks to the fast decay of integrand $I_{j l}(w, y)$ in any direction of plane $(w, y)$, the integrals upon $w$ and $y$ can be exchanged. Then in the integral upon $w$, we make the variable change $w \rightarrow \eta$ with

$$
\eta=(1+2 y)^{1 / 2}\left(w-\frac{y(1+y)}{1+2 y}+\frac{i k y}{\epsilon(1+2 y)}\right) .
$$

This provides

$$
\begin{aligned}
A_{j l}(k ; z)= & \frac{\epsilon}{\sqrt{2 \pi}} \int_{0}^{\infty} \mathrm{d} y \frac{1}{(1+2 y)^{1 / 2}} \\
& \times \exp \left(-(z+1) y-\epsilon^{2} \frac{y^{3}(2+y)}{6(1+2 y)}-\frac{k^{2} y^{2}}{2(1+2 y)}-\frac{i k \epsilon y^{2}(1+y)}{1+2 y}\right) \\
& \times \int \mathrm{d} \eta \exp \left(-\frac{\eta^{2}}{2}\right)\left(\frac{\eta}{1+2 y)^{1 / 2}}+\frac{\epsilon y(1+y)}{1+2 y}-\frac{i k y}{1+2 y}\right)^{j} \\
& \times\left(\frac{\eta}{1+2 y)^{1 / 2}}-\frac{\epsilon y^{2}}{1+2 y}-\frac{i k y}{1+2 y}\right)^{l}
\end{aligned}
$$


The integral upon $\eta$ can be easily performed, thanks to the simple dependence of the corresponding integrand with respect to $\eta$, namely a Gaussian times a polynomial. The result is a combination of algebraic functions of $y$ with coefficients which reduce to positive integer powers of $k$. Thus, the remaining integral upon $y$ does converge for any complex value of $k$ and $z$, thanks to the presence of factor

$$
\exp \left(-\epsilon^{2} \frac{y^{3}(2+y)}{6(1+2 y)}\right)
$$

which ensures a fast integrable decay of the integrand when $y \rightarrow \infty$. That fast decay guarantees that $A_{j l}(k ; z)$ is an entire function of both complex variables $k$ and $z$.

Integral representation (C1) can be specified to $k=0, j=0,1$ and $l=0,1$. This provides useful expressions for functions $A_{00}(z), A_{10}(z), A_{01}(z)$ and $A_{11}(z)$ which are analogous to formula (54) for $\Delta(z)$. That formula is derived as follows. First, we compute $\mathrm{d} \Delta / \mathrm{d} z$ from expression (A10) specified to $k=0$ in terms of functions $A_{j l}(z)$ and of their derivatives with respecto $z$. Using the integral representations (A6), such derivatives are then expressed in terms of the $A_{j l}(z)$ 's by combining differentiation under the integral sign and integration by parts. This allows us to infer that $\Delta(z)$ is the solution of the first order differential equation

$$
\frac{\mathrm{d} \Delta}{\mathrm{d} z}-\Delta=3 \epsilon \frac{\mathrm{d} A_{00}}{\mathrm{~d} z}-\epsilon A_{00}
$$

with the boundary condition at infinity $\Delta(z) \rightarrow 0$ when $z=x \rightarrow \infty$. A straightforward application of the constant-variation method leads to

$$
\Delta(z)=3 \epsilon A_{00}(z)-2 \epsilon \exp (z) \int_{z}^{\infty} \mathrm{d} z^{\prime} \exp \left(-z^{\prime}\right) A_{00}\left(z^{\prime}\right)
$$

Eventually, we use the above integral representation of $A_{00}\left(z^{\prime}\right)$ into expression (C3), and we exchange integrals upon $z^{\prime}$ and $y$ thanks to absolute convergence. Since the dependence in $z^{\prime}$ reduces to simple exponential factor $\exp \left(-z^{\prime}(1+y)\right)$, the integral upon $z^{\prime}$ is readily done, and this eventually leads to formula (54).

[1] A. Gervois, J. Piasecki, J.Stat.Phys. 42:1091-1102 (1986)

[2] J. Piasecki, J.Stat.Phys. 30:185 (1983). 
[3] J. Piasecki, Phys.Lett.A 114:245-249 (1986)

[4] J. Piasecki, R. Soto, Physica A 369:379-386 (2006)

[5] A.V. Bobylev, J.A. Carillo, I.M. Gamba, J.Stat.Phys. 98:743-773 (2000)

[6] A.V. Bobylev, C. Cercignani, J.Stat.Phys. 106:1019 (2002)

[7] Ph.A. Martin, J. Piasecki, J.Phys.A:Math. Theor. 40:361-369 (2007)

[8] G.E. Uhlenbeck, G.W. Ford, E.W. Montroll, Lectures in statistical physics (American Mathematical Society, 1963)

[9] M.H. Ernst and J.R. Dorfman, J.Stat.Phys. 12:311-359 (1975)

[10] M.H. Ernst, J.Stat.Phys. 34:issues 5,6 (1984)

[11] F. Coppex, M. Droz, E. Trizac, Phys.Rev.E 72: 021105 (2005) 